\title{
A DFT-based model to the interpretation of DC conductivity in transition metals doped zinc phosphate glass
}

\author{
C Maghni ${ }^{1,2}$ and M Kharroubi ${ }^{1 *}$ \\ ${ }^{1}$ Physico-chemistry of Materials and Environment Laboratory, Ziane Achour University of Djelfa, BP 3117, Djelfa, Algeria \\ ${ }^{2}$ Department of Physics, Faculty of Science, Med Boudiaf University-M'Sila, Bp 166, 28000 M'sila, Algeria
}

Received: 15 August 2020 / Accepted: 13 April 2021 / Published online: 7 May 2021

\begin{abstract}
Ternary zinc-sodium-phosphate glasses doped with transition metal of the composition $\mathrm{Na}_{2} \mathrm{M}_{x} \mathrm{Zn}_{1-x}$ $\mathrm{P}_{2} \mathrm{O}_{7}(x=0,1,2$ and $5 \mathrm{~mol} \%$ ) (where $\mathrm{M}=\mathrm{Ni}, \mathrm{Cu}$ and $\mathrm{Co}$ ) were prepared by the traditional quenching method. The ac conductivity measurements at different temperatures for the prepared glasses have been investigated, and the activation energy for dc conduction has been determined in each transition metal doped sample. The results showed that the evolution of the activation energy of the conductivity depends on the nature of the dopant ions. A model based on formal density functional theory concept in which the electrical charge exchanged between the transition-metal cations and the surrounding material surface is proposed. The outcome is a "simplified" formula which allows us to explain the evolution of the ionic dc conductivity activation energy as a function of the doped ion in interaction with the cation and the surface.
\end{abstract}

Keywords: Phosphate glass; Model; Conductivity; Transition metals; Density functional theory; Complex impedance spectroscopy

\section{Introduction}

Glasses containing transition metal ions (TMI) are of permanent interest due to their optical, electrical and structural properties [1-4]. The study of the temperature dependence of electrical transport is very much crucial in understanding the charge transport mechanisms through transition metal oxides (TMO) doped glasses. The conduction mechanism in transition metal oxides doped glass has been supposed to be the small polaron hoping (SPH) $[5,6]$. In the polaron model, a conduction electron localizes at a site, or atom, in the system and causes a lattice distortion which stabilizes (traps) the localized electron. This electron migrates from site to site, via a hopping mechanism, primarily through thermal motion [7, 8]. Great attention has been given to investigate the effect of the transition metal oxides on the electronic transport properties of glasses [9], but very few studies have been reported about phosphate glasses containing TMO. It has been suggested by Sayer et al. that the conduction processes in phosphate glasses containing transition metal ions suggest that a polaron model is

*Corresponding author, E-mail: kharroubi3@yahoo.fr generally applicable, with some evidence that hopping occurs in the adiabatic regime [10].

The purpose of the present study is to measure the dc electrical conductivity of doped zinc phosphate glass with different transition metals ions, probed by means of complex impedance spectroscopy (CIS) measurements. First, the dc conductivity of each transition metal doped sample was measured at different temperatures to determine the activation energy $\Delta E_{\mathrm{dc}}$. Then, a model, which is based on the density functional theory approach, was proposed to fit the evolution of the activation energy for the dc conductivity with the various transition metals cations in phosphate glasses.

\section{Experimental details}

\subsection{Sample preparation}

The glasses used in this study are obtained following the same preparation route as that described in [11]. Briefly, the appropriate amounts of high purity chemicals powders $(\geq 99.9 \%) \quad \mathrm{NH}_{4} \mathrm{H}_{2} \mathrm{PO}_{4} \quad(\geq 99.9 \%, \quad \mathrm{POCH}), \quad \mathrm{Na}_{2} \mathrm{CO}_{3}$ ( $\geq 99.5 \%$, SIGMA-ALDRICH), $\mathrm{ZnO}(\geq 99.9 \%$, RIEDEL $)$ 
Table 1 Molar composition of the prepared glasses

\begin{tabular}{|c|c|c|c|c|c|c|c|c|c|c|}
\hline \multirow{2}{*}{$\begin{array}{l}\text { Glass } \\
x \text { mol \% }\end{array}$} & \multicolumn{4}{|c|}{ NZPO 1} & \multicolumn{3}{|c|}{ NZPO 2} & \multicolumn{3}{|c|}{ NZPO 3} \\
\hline & 0 & 1 & 2 & 5 & 1 & 2 & 5 & 1 & 2 & 5 \\
\hline $\mathrm{P}_{2} \mathrm{O}_{5}$ & 50.00 & 50.00 & 50.00 & 50.00 & 50.00 & 50.00 & 50.00 & 50.00 & 50.00 & 50.00 \\
\hline $\mathrm{ZnO}$ & 25.00 & 24.75 & 24.50 & 23.75 & 24.75 & 24.50 & 23.75 & 24.75 & 24.50 & 23.75 \\
\hline $\mathrm{Na}_{2} \mathrm{O}$ & 25.00 & 25.00 & 25.00 & 25.00 & 25.00 & 25.00 & 25.00 & 25.00 & 25.00 & 25.00 \\
\hline $\mathrm{NiO}$ & 00.00 & 00.25 & 00.50 & 01.25 & 00.00 & 00.00 & 00.00 & 00.00 & 00.00 & 00.00 \\
\hline $\mathrm{CoO}$ & 00.00 & 00.00 & 00.00 & 00.00 & 00.25 & 00.50 & 01.25 & 00.00 & 00.00 & 00.00 \\
\hline $\mathrm{CuO}$ & 00.00 & 00.00 & 00.00 & 00.00 & 00.00 & 00.00 & 00.00 & 00.25 & 00.50 & 01.25 \\
\hline
\end{tabular}

$\mathrm{Ni}\left(\mathrm{NO}_{3}\right)_{2} \quad(\geq 99.9 \%, \quad$ SIGMA-ALDRICH $), \quad \mathrm{Cu}\left(\mathrm{NO}_{3}\right)_{2}$ $(\geq 99.9 \%, \quad$ SIGMA-ALDRICH $), \quad \mathrm{Co}\left(\mathrm{NO}_{3}\right)_{2} \quad(\geq 99.9 \%$, SIGMA-ALDRICH) were mixed in an agate mortar. The mixture was progressively heated in a platinum crucible from 298 to $633 \mathrm{~K}$ in order to remove both ammonia and water. After grinding, the powders were heated at $993 \mathrm{~K}$ for two days in order to reach the complete departure of carbon dioxide. Then, the mixture was melted $6 \mathrm{~h}$ at $1093 \mathrm{~K}$. Finally, the glass obtained was maintained $12 \mathrm{~h}$ at $573 \mathrm{~K}$ to improve its mechanical stability. Basing on the transition metal ion of dopant in the zinc phosphate glass, the samples were designated as, NZPO 1 (nickel), NZPO 2 (cobalt) and NZPO 3 (copper), respectively. The sample identification and the respective molar composition are reported in Table 1.

\subsection{Conductivity measurements}

Dc conductivity is extracted from ac conductivity signal recorded at various constant measurement temperatures $T$ in the $10^{-2}-10^{6} \mathrm{~Hz}$ frequency range from a Novocontrol Broadband Dielectric Spectrometer (BDS 4000) [12]. For each temperature, the sample is maintained $30 \mathrm{~min}$ before measurement in order to reach thermal equilibrium. From prior characterizations, the glasses as fine powder were compacted to a disk-shape pellet (diameter $13 \mathrm{~mm}$, thickness $\approx 1 \mathrm{~mm}$, weight $0.3 \mathrm{~g}$ ). Then, both sides of the glass samples were coated with the gold electrodes using Sputter Coater SC7620 in order to ensure the electrical connection. For each temperature, the sample is maintained $30 \mathrm{~min}$ before measurement to reach thermal equilibrium.

\subsection{Results}

Electrical conductivity is a principal factor that reveals vital information about the properties of electrical dynamic and transport phenomenon in glass materials.

Figure 1 shows the change in ac conductivity of the reference sample (no doped sample) of some selected samples with changing frequencies for different temperatures.

Each transition metal ion sample showed a similar behavior. In each case, we noted that the ac conductivity was the sum of two contributions [13].

$\sigma_{\mathrm{ac}}(\omega)=\sigma_{\mathrm{dc}}+\sigma^{\prime}(\omega)$,

where $\sigma_{\mathrm{dc}}$ is frequency independent and it is identified with continuous conductivity and the second term is the purely dispersive component of the polarization conductivity, depending on the frequency $\omega(\omega=2 \pi f$ is the angular frequency), in general, given as $\sigma^{\prime}(\omega, T)=A(T) \omega^{n}$. Here, factor $\mathrm{A}$ is a constant which determines the strength of polarizability, and exponent $n(n \leq 1)$ that represents the degree of interaction between mobile ions and the lattices around them. It can be said that the dependence of the conductivity as a function of the temperature varies with the transition metal ions. To determine the activation energy, $\Delta E_{\mathrm{dc}}$, which characterizes the dc conductivity of each sample of phosphate glass using the Nernst-Einstein equation.

$\sigma_{\mathrm{dc}}=\frac{\sigma_{0}}{T} \exp \left(\frac{-\Delta E_{\mathrm{dc}}}{K T}\right)$,

where $\Delta E_{\mathrm{dc}}$ is the activation energy, $\sigma_{0}$ a pre-exponential factor and $k_{\mathrm{B}}$ the Boltzmann constant. Arrhenius plot of the whole sample glasses series is reported in Fig. 2 (1 mol\%) where it can be seen that dc conductivity strongly depends on the considered sample and that all samples follow the thermal behavior.

Figure 3 shows the activation energy as a function of the TMI for various ratios. Whatever the TMI, we can observe that the $\Delta E_{\mathrm{dc}}$ is lower for glass doped with $\mathrm{Co}^{2+}$ and $\mathrm{Ni}^{2+}$ than the one doped with $\mathrm{Cu}^{2+}$. The higher values of $\mathrm{dc}$ energy, $\Delta E_{\mathrm{dc}}$, observed for glass doped with copper is likely due to the electronic conductivity which becomes dominant. This induces that the small polarons hope easily between $\mathrm{Cu}^{+}$and $\mathrm{Cu}^{2+}$ [14]. A more detailed discussion about these values and their evolution was given in our previous paper [15]. 


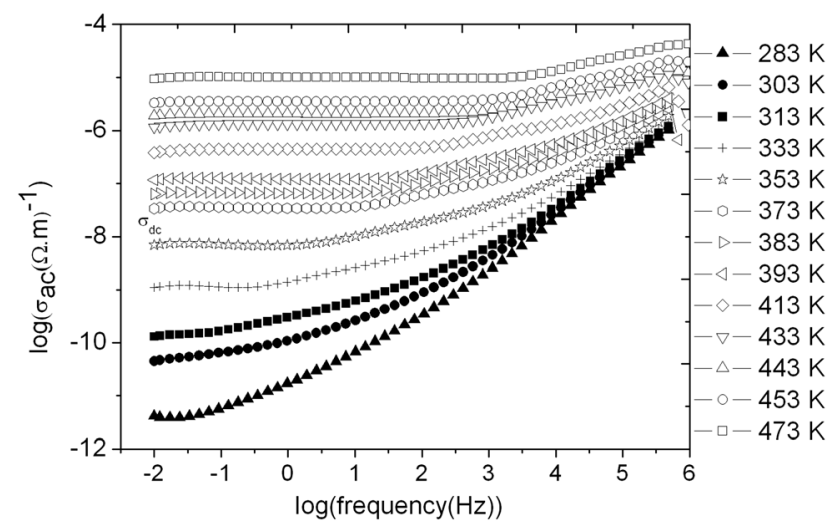

Fig. 1 The relation between the logarithm of the conductivity $\sigma_{\mathrm{ac}}$ and frequency at different temperatures for the undoped sodium zinc phosphate glass

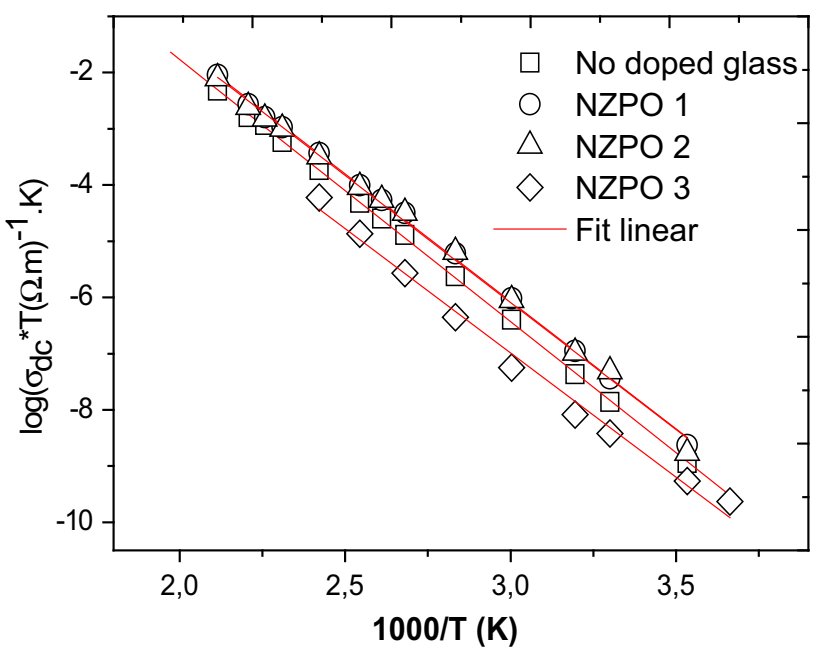

Fig. 2 Variation of dc conductivity, $\sigma_{\mathrm{dc}}$ versus 1000/T doped of $1 \mathrm{~mol} \%$ of dopant. Symbols represent the experimental data. The straight lines represent the fits obtained from Eq. (2)

\section{The model}

One of the most relevant traits of the density functional language is its suitability to calculate the extraction energy $\Delta E_{\text {hop }}$ of a cation out of its site. Parallel to the development of quantum-chemical models to approach the HohenbergKohn equation [16], Parr developed the so-called "conceptual DFT" in the late 1970s and early 1980s [17]. Conceptual DFT is a DFT-subfield in which one tries to extract from the electron density relevant concepts and principles that make it possible to understand and predict the chemical behavior of a molecule. Parr and co-workers, and later a large community of theoretical chemists, have been able to give precise definitions for chemical concepts which had already been known and used for many years in various branches of chemistry; electronegativity is the most noticeable example, thus providing their calculations with

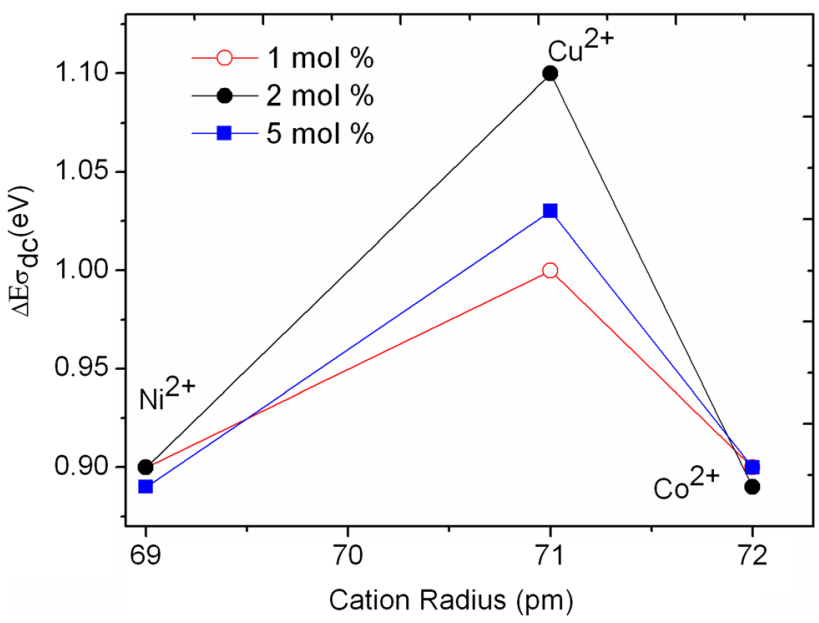

Fig. 3 Variation of activation energy as a function of ionic radius $R_{\mathrm{C}}$ at $373 \mathrm{~K}$ for $\mathrm{Na}_{2} \mathrm{M}_{x} \mathrm{Zn}_{1-x} \mathrm{P}_{2} \mathrm{O}_{7}$ glasses doped with $\mathrm{Ni}^{2+}$ (NZPO 1), $\mathrm{Co}^{2+}$ (NZPO 2) and $\mathrm{Cu}^{2+}$ (NZPO 3) at 1,2 and $5 \mathrm{~mol} \%$, respectively. Full lines are just to guide the eyes

a quantitative explanation for the experimental evolution of $\Delta E_{\text {bond }}$ in some oxides and hence to quantitatively determine the relative weights of the electrostatic, covalent and polarization contributions to the energy of the transition metal bond.

First, it must be recalled that $\Delta E_{\sigma_{\mathrm{dc}}}$ can be formally seen as the sum of two contributions

$\Delta E_{\sigma_{\mathrm{dc}}}=\Delta E_{\mathrm{bond}}+\Delta E_{\mu}$,

where $\Delta E_{\text {bond }}$ is the cation-oxide bonding energy, i.e., the energy necessary for extracting a given cation from its potential minimum, and $\Delta E_{\mu}$ is the energy related to the cation migration over a long distance. This well-known relation suggests that the interpretation of the polarization and the conductivity may have common bases. In this contribution, it will be shown how a very simple modeling of the release of the cations leads to a relatively general law explaining the experimental results. This modeling takes into account, with a few approximations, the density functional theory [18] to calculate the extraction energy $\Delta E_{\text {bond }}$ of a cation out of its site. The latter is the sum of three independent contributions [19]: an electrostatic term, $\Delta E_{\text {Elec }}$, a covalence term, $\Delta E_{\mathrm{cov}}$, and a term associated with the polarizability of each species, $\Delta E_{\mathrm{pol}}$. An evaluation of $\Delta E_{\text {bond }}$ can be made using the density functional theory [20] and the electronegativity equalization method [21]. The simplified expressions of each energy term can be made when the critical parameter is the ionic radius, $R_{\mathrm{C}}$, of a given transition metal. However, these simulations are hampered on solid oxides as complex and as large as clay minerals and glassy oxides as the computational work they would require is too intensive. Consequently, the purpose of this work is to propose crude but realistic simplifications 
that aim to build a convenient method for fitting the experimental data and for acquiring a deeper knowledge of the nature of cation-oxide bonds in these solids.

The formation of a bond of the type of the chemical pseudo-reaction, i.e., between two isolated ions, is done by means of a charge transfer $\delta N$. This pseudo-chemical reaction can also be represented by a thermally activated dissociation [22].

In this work we have tried to calculate the evolution of the variation of electronic cloud energy of a species that belongs to the studied surface and that can be expressed by a series of second order of two variables: first, during this elementary displacement, its electronic charge $N_{\mathrm{c}}$ becomes $N_{\mathrm{c}}+\delta N_{\mathrm{c}}$. Second, the modification of the electric potential that surrounds the system becomes:

$$
\begin{aligned}
E= & E_{0}+\left(\frac{\partial E}{\partial N}\right)_{V_{G}} \delta N+\left(\frac{\partial E}{\partial V}\right) \delta N_{G}+\frac{1}{2}\left(\frac{\partial^{2} E}{\partial N^{2}}\right)(\delta N)^{2} \\
& +\frac{1}{2}\left(\frac{\partial^{2} E}{\partial V_{G}^{2}}\right)\left(\delta V_{G}\right)^{2}+\left(\frac{\partial^{2} E}{\partial N \partial V_{G}}\right) \partial N \partial V_{G},
\end{aligned}
$$

where $\left(\frac{\partial E}{\partial V_{G}}\right)=\rho$ is the electronic density, and $\left(\frac{\partial E}{\partial N}\right)_{V_{s}}=\mu$ is the chemical potential. The derivate of the electronic energy relative to the number of electrons and $\left(\frac{\partial^{2} E}{\partial N^{2}}\right)=\eta$ is the hardness which is the second derivative of the energy.

Our aim is to determine the elementary variation of the energy of the electron cloud of the cation, through a second-order expansion [23].

$$
\begin{aligned}
E_{c}= & \mu_{0, c} \delta N+\int_{0}^{\infty} \rho_{c} \delta V_{G} \mathrm{~d} r+\eta_{c}(\delta N)^{2}+\delta N \int_{0}^{\infty} f_{c}(r) \delta V_{G} \mathrm{~d} r \\
& +\iint\left(\frac{\partial \rho_{c}}{\delta V_{G}}\right) \delta V_{G}(r) \delta V_{G}\left(r^{\prime}\right) \mathrm{d} r \mathrm{~d} r^{\prime},
\end{aligned}
$$

Using the same equation for the glass surface surrounding the cation, and assuming that the energy barrier corresponds to the energy difference between the energy of the cation trapped at the surface and that when it is free, we can write

$$
\begin{aligned}
\delta(\Delta E)= & \left(\mu_{0, c}-\mu_{0, G}\right) \delta N+\int_{0}^{\infty} \rho_{c} \delta V_{G} \mathrm{~d} r+\int_{0}^{\infty} \rho_{G} \delta V_{c} \mathrm{~d} r \\
& +\left(\eta_{c}+\eta_{G}\right)(\delta N)^{2}+\delta N\left[\int_{0}^{\infty} f_{c} \delta V_{G} \mathrm{~d} r+\int_{0}^{\infty} f_{G} \delta V_{c} \mathrm{~d} r\right] \\
& +\iint\left(\frac{\partial \rho_{c}}{\delta V_{G}}\right) \delta V_{G}(r) \delta V_{G}\left(r^{\prime}\right) \mathrm{d} r \mathrm{~d} r^{\prime} \\
& +\iint\left(\frac{\partial \rho_{G}}{\delta V_{c}}\right) \delta V_{c}(r) \delta V_{c}\left(r^{\prime}\right) \mathrm{d} r \mathrm{~d} r^{\prime},
\end{aligned}
$$

The energy associated with the extraction of cation out of its site always shows an electronic component $\delta \mathrm{E}$, with the sum of three contributions [24, 25].

$\delta E=\delta E_{\text {Elec }}+\delta E_{\mathrm{Cov}}+\delta E_{\mathrm{pol}}$,

Each of these terms is the sum of the contribution of the cation and of the site. Calculations and approximations that have been detailed elsewhere [26] lead to the following expressions:

$$
\begin{aligned}
& \delta E_{\text {Elec }}=\int_{0}^{\infty} \rho_{C}(r) \delta V_{C, \text { ext }}(r) \mathrm{d} r+\int_{0}^{\infty} \rho_{G}(r) \delta V_{G, e x t}(r) \mathrm{d} r \\
& \delta E_{\mathrm{cov}} \\
& \quad \int_{0}^{\infty}\left[\left(\frac{\partial \rho_{C}(r)}{\partial N}\right) \delta V_{C, \text { ext }}(r)-\left(\frac{\partial \rho_{G}(r)}{\partial N}\right) \delta V_{G, e x t}(r)\right] \mathrm{d} r+\cdots+\frac{1}{4\left(\eta_{C}+\eta_{G}\right)} \\
& \quad \times\left(\int_{0}^{\infty}\left[\left(\frac{\partial \rho_{C}(r)}{\partial N}\right) \delta V_{C, \text { ext }}(r)-\left(\frac{\partial \rho_{G}(r)}{\partial N}\right) \delta V_{G, \text { ext }}(r)\right] \mathrm{d} r\right)^{2},
\end{aligned}
$$

and

$$
\begin{aligned}
\delta E_{\mathrm{pol}}= & \iint\left(\frac{\partial \rho_{C}(r)}{\partial V_{C, \mathrm{ext}}\left(r^{\prime}\right)}\right) \delta V_{C, \text { ext }}(r) \delta V_{C, \text { ext }}\left(r^{\prime}\right) \mathrm{d} r \mathrm{~d} r^{\prime} \\
& +\iint\left(\frac{\partial \rho_{G}(r)}{\partial V_{G, \text { ext }}\left(r^{\prime}\right)}\right) \delta V_{G, \text { ext }}(r) \delta V_{G, \text { ext }}\left(r^{\prime}\right) \mathrm{d} r \mathrm{~d} r^{\prime},
\end{aligned}
$$

In these equations, the subscripts $\mathrm{C}$ and $\mathrm{G}$ refer to the transition metal cation and the solid oxide (Glass) grid, respectively. $\rho(r)$ is the electron density and $\mu_{0}$ is the chemical potential of the standard electrons of the isolated species. However, further approximations can be introduced and simplified expressions for each energy term are proposed. These expressions are given as a function of the transition metal cations radius $R_{\mathrm{C}}$.

\subsection{The electrostatic term $\Delta E_{\text {elec }}$}

It is appropriate to assume that the electrostatic term is proportional to the inverse of the cation radius, the proportionality coefficient is related to the Madelung constant, which is independent of transitional metal cations and depends solely on the rigid grid which is not affected by the movement of the charge, as in the Anderson and Stuart model (A-S model) [27]. Provided that the charges on the cation and the framework are of opposite sense, we can write

$\Delta E_{\text {elec }} \propto \frac{1}{R_{\mathrm{C}}+R_{\mathrm{O}}}$,

where $R_{\mathrm{O}}$ is the radius of the $\mathrm{O}^{2-}$ ion with its charge of $-2 e\left(R_{\mathrm{O}}=140 \mathrm{pm}\right)$. The evolution of this energy is reported in Fig. 4(a). 

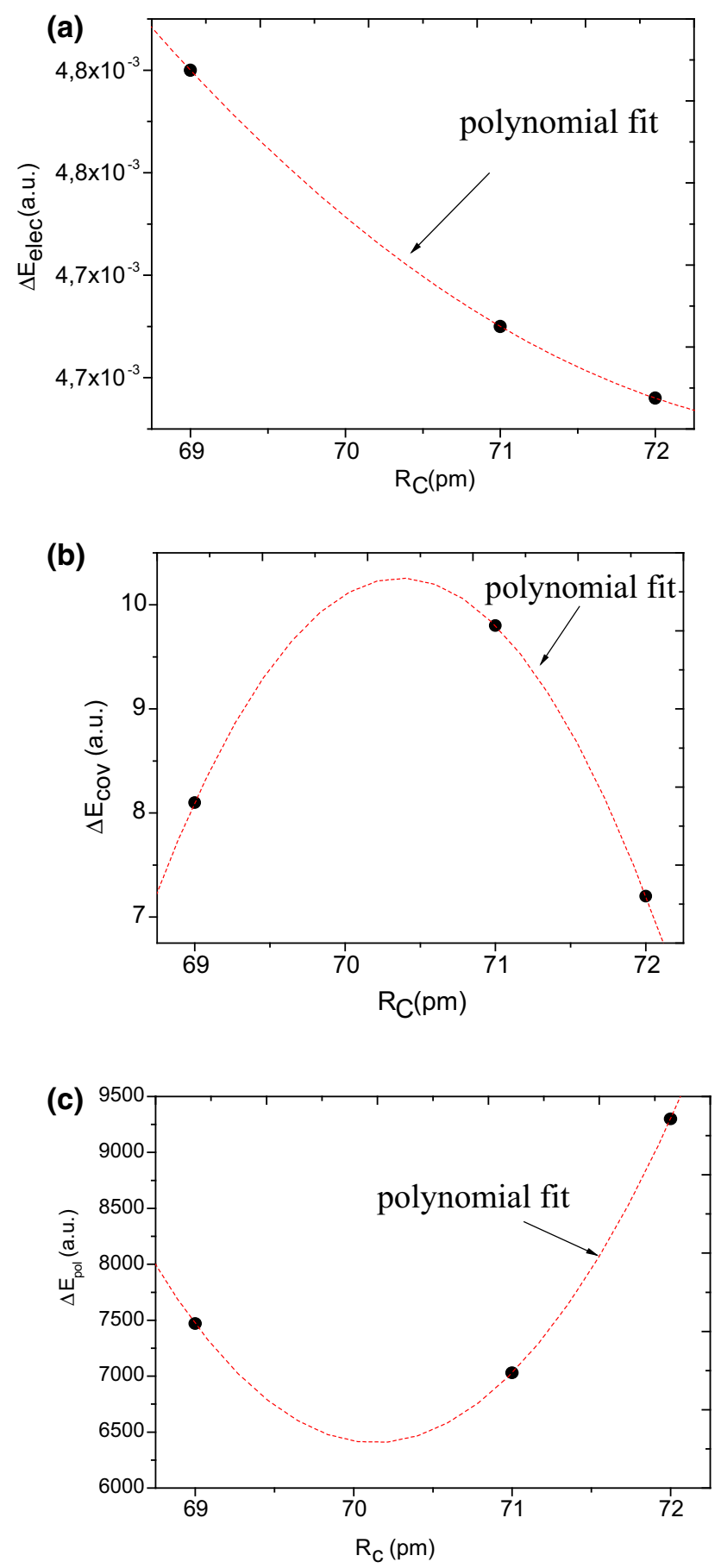

Fig. 4 Variation of the (a) electrostatic, (b) covalent, and (c) polarization energy terms versus cation radius. Symbols represent the data calculated from Eqs. (11), (12), and (14) and reported in Table 3 for (b), and in Table 4 for (c). The dashed lines are the polynomial fits corresponding to Eqs. (11), (13), and (15)

\subsection{The covalent term $\Delta E_{\text {cov }}$}

This term accounts for the exchange of charges between the site and the cation. Therefore, it depends on both the nature of the solid grid cations, $\mathrm{C}^{+}$, and the network itself,
$\mathrm{G}^{-}$.The cation is trapped in a potential well, the characteristics of which are determined by the different charges of the polyanionic network which surround it Eq. (9).

We, therefore, used the values of $\mu_{0, \mathrm{C}}$ and $\eta_{\mathrm{C}}$ which are offered by Pearson and Parr [28] taking into account the ionization energy of the corresponding elements (see Table 2). Some values of $\mu_{0, \mathrm{G}}$ and $\eta_{\mathrm{G}}$ have already been estimated from emulation in some modeled oxides and zeolites [29], where it was found to differ only slightly from one oxide material to another oxide material. However, an estimate of $\mu_{0, \mathrm{G}}$ and $\eta_{\mathrm{G}}$ can be obtained from the principle proposed by both Sanderson and Aniya [30, 31]. The three terms of Eq. (9) reported in Table 3 are calculated with $\mu_{0, G}=5.84 \mathrm{eV}$ and $\eta_{G}=4.9 \mathrm{eV}$.

First, it can be observed that the values of the third column are constant for different transition metal cations. Second, the values of the first column are much greater than those of the second and third columns. Assuming that the integral terms in Eq. (9) are negligible and not taking into account factors local in the nature of the chemical shackle, this is to simplify the expression. Consequently, this assumption is the only way to simplify the expression and hence to provide a qualitative evaluation of the energy evolution for dc conductivity. $\Delta E_{\mathrm{cov}}$ can then be simplified as follows

$\Delta E_{\mathrm{cov}} \propto \frac{\left[\mu_{0, C}-\mu_{0, G}\right]^{2}}{4\left(\eta_{C}+\eta_{G}\right)}$,

With this model, the value of $\Delta E_{\mathrm{cov}}$ changes as the transition metal cation radius, $R_{C}$, and its evolution can be represented by a second order polynomial function (see Fig. 4b) for which the characteristic parameters are obtained by a nonlinear least-squares fitting procedure. The fit is represented in Fig. 4(b) and corresponds to the following polynomial function

$\Delta E_{\mathrm{cov}} \approx-1.15 R_{C}^{2}+1.62 \times 10^{2} R_{C}-5.68 \times 10^{3}$,

The fitting parameters were obtained for $\Delta E_{\mathrm{cov}}$ in units of $\mathrm{eV}$ and for $R_{C}$ in units of $\mathrm{pm}$.

\section{The polarization term $\Delta E_{\text {pol }}$}

The polarization energy (see Eq. (10)) is the sum of two participants of the same shape related to the transition metal cations and the oxide network. In order to estimate the polarization energy of the cations, we have defined a simple parameter which accounts, a priori, for the sensitivity of the electron cloud of anion, to a modification of the acting external potential, $V_{\text {ext }}$. It is known that in this approach, the electronic surface density has a very important effect, so the higher the surface density is, the 
Table 2 Corresponding values of the electronic chemical potential and the hardness for each atom and for the various test cases oxides

\begin{tabular}{llllll}
\hline Element & $\mu_{0}[23](\mathrm{eV})$ & $\eta[23](\mathrm{eV})$ & Cation & $\mu_{O, C}[23](\mathrm{eV})$ & $\eta_{\mathrm{C}}[23](\mathrm{eV})$ \\
\hline $\mathrm{Na}$ & 2.85 & 2.30 & $\mathrm{Ni}^{2+}$ & 26.67 & 8.50 \\
$\mathrm{Zn}$ & 4.45 & 4.94 & $\mathrm{Cu}^{2+}$ & 28.56 & 8.27 \\
$\mathrm{P}$ & 5.62 & 4.88 & $\mathrm{Co}^{2+}$ & 25.28 & 8.22 \\
$\mathrm{O}$ & 7.54 & 6.08 & & & \\
\hline
\end{tabular}

Table 3 Comparison of the variation of preintegral terms of Eq. (9), depending on the nature of the transition metal ions

\begin{tabular}{llll}
\hline Cations & $\frac{\left(\mu_{0, \mathrm{C}}-\mu_{0, \mathrm{G}}\right)^{2}}{4\left(\eta_{\mathrm{C}}+\eta_{\mathrm{G}}\right)}(\mathrm{eV})(\mathrm{eV})$ & $\frac{\left(\mu_{0, \mathrm{C}}-\mu_{0, \mathrm{G}}\right)}{2\left(\eta_{\mathrm{C}}+\eta_{\mathrm{G}}\right)}$ & $\frac{1}{4\left(\eta_{\mathrm{C}}+\eta_{\mathrm{G}}\right)}\left(\mathrm{eV}^{-1}\right)$ \\
\hline $\mathrm{Ni}^{2+}$ & 8.1 & 0.777 & 0.019 \\
$\mathrm{Cu}^{2+}$ & 9.8 & 0.863 & 0.019 \\
$\mathrm{Co}^{2+}$ & 7.2 & 0.741 & 0.019 \\
\hline
\end{tabular}

Table 4 Compute values of the $\mathrm{SED}^{-1}$ in $\mathrm{pm}^{2}$ from the radius [31], and the number of valence electrons for different transition metals cations

\begin{tabular}{llll}
\hline Cations & Radius $(\mathrm{pm})$ & Number of valence $\mathrm{e}^{-}$ & $\mathrm{SED}-1\left(\mathrm{pm}^{2}\right)$ \\
\hline $\mathrm{Ni}^{2+}$ & 69 & 8 & $7.47 \times 103$ \\
$\mathrm{Cu}^{2+}$ & 71 & 9 & $7.03 \times 103$ \\
$\mathrm{Co}^{2+}$ & 72 & 7 & $9.30 \times 103$ \\
\hline
\end{tabular}

lower the influence of external potentials is, and therefore, the participation of electrostatic and covalent terms in the term polarization energy is negligible, and on this basis, $\Delta E_{\text {pol }}$ can be limited to the cation participation, $\Delta E_{C, \text { pol }}$ only. However, it is thought that this problem is similar to the determination of the influence of an electrostatic potential on an electrically charged sphere. A simple model has also been proposed [25] where $\Delta E_{C, \text { pol }}$ is supposed to be inversely proportional to a parameter termed the "Surface Electronic Density" (SED), which is defined as

$\Delta E_{C, \mathrm{pol}} \propto \mathrm{SED}^{-1}=\frac{4 \pi R_{C}^{2}}{N_{C, \mathrm{ext}}}$,

where $N_{C \text {.ext }}$ denotes the number of valence electrons.

The results, thus, obtained are reported in Table 4 . We then assumed that the evolution of the contribution of the polarization energy, $\Delta E_{C \text {,pol }}$, to the hopping energy of the cation, could be represented, in a first approximation, by values proportional to the SED reported in Table 4, and plotted as a function of $R_{C}$ in Fig. 4(c). It can be seen that the values of $\mathrm{SED}^{-1}$ are very close. As for $\Delta E_{\text {cov }}$, we can represent $\Delta E_{\mathrm{pol}}$ by a polynomial function of second order, whose parameters are determined from a least squares fit procedure. The fit to our data is shown in Fig. 4(c) and corresponds to the following polynomial function

$\Delta E_{\mathrm{pol}} \approx 830 R_{C}^{2}-11.64 \times 10^{4} R_{C}+4.09 \times 10^{6}$,

The fitting parameters are obtained for $\mathrm{SED}^{-1}$ in units of $\mathrm{pm}^{2}$ and $R_{C}$ in units of $\mathrm{pm}$.

\section{Fitting of the experimental data}

The dependence of $\Delta E_{\mathrm{dc}}$ on $R_{C}$ is then given by the summation of each of the energy derivative terms

$\Delta E_{\mathrm{bond}}=x_{\text {elec }} \Delta E_{\text {elec }}+x_{\mathrm{cov}} \Delta E_{\mathrm{cov}}+x_{\mathrm{pol}} \Delta E_{\mathrm{pol}}$,

The adjustable parameters, $\mathrm{x}_{i}$, can be obtained from a fit of the experimental data with a linear combination of the derivative functions, $\Delta E_{\text {elec }}$ (Eq. (11)), $\Delta E_{\text {cov }}$ (Eq. (13)) and $\Delta E_{\mathrm{pol}}$ (Eq. (15)) for all samples. The present parameters correspond to the product of the weight of each contribution by the coefficient of proportionality that relates the measured energy values to their associated, simplified expressions (Eqs. (13) and (15)). Consequently, the parameter $x_{\text {cov }}$ which is dimensionless, yields an energy value, while $x_{\text {elec }}$ and $x_{\text {pol }}$ are in units of $\mathrm{eV} \mathrm{pm}$ and $\mathrm{eVpm}^{-2}$, respectively. However, it is possible to make a comparison between the values obtained from the various test-case transition-metals ions doped zinc phosphate glasses. In a first step, the nonlinear least-squares procedure is achieved for a freely adjustable set of parameters $x_{\text {elec }}, x_{\mathrm{cov}}$ and $x_{\mathrm{pol}}$ that complies with the imposed constraints. This key result shows that the ionic conductivity of the investigated transition metal doped phosphate glass samples depends on the three electrostatic, covalent and polarization terms. Then, we fix to zero one or two of these parameters to check whether their contribution noticeably improves the quality of the fit. If it does not, we then consider that the parameter $x_{\text {elec }}$ need not be taken into account. In that case, the fit leads to constraining the parameter $x_{\text {cov }}$ to positive while $x_{\text {pol }}$ remains negative (see Table 5). It means, for example, that the deformation, i.e., the polarizability of the cation as well as the covalency of 
Table 5 Values parameters obtained from the nonlinear least-squares fit of the experimental data of all glasses samples

\begin{tabular}{llll}
\hline$x \mathrm{~mol} \%$ & $x_{\mathrm{elec}}(\mathrm{eV} \cdot \mathrm{pm})$ & $x_{\mathrm{cov}}$ & $x_{\mathrm{pol}}\left(\mathrm{eV} \cdot \mathrm{pm}^{-2}\right)$ \\
\hline $1 \%$ & 0 & $2 \times 10^{-5}$ & $-6 \times 10^{-5}$ \\
$2 \%$ & 0 & $2 \times 10^{-5}$ & $-11 \times 10^{-5}$ \\
$5 \%$ & 0 & $2 \times 10^{-5}$ & $-8 \times 10^{-5}$ \\
\hline
\end{tabular}

$x_{\text {elec }}, x_{\text {cov }}$ and $x_{\text {pol }}$ correspond to the results obtained when the constraints in the fitting procedure were released

the ionic bond, plays a significant role in the transition metal-glass bond.

Figure 5 clearly confirms the monotonous evolution of the activation energy of the dc conductivity, determined on transition-metals ions doped zinc phosphate glasses $(2 \mathrm{~mol}$ $\%$ ), which reveals that $\Delta E_{\text {cov }}$ is preponderant. It was also found that the evolution is similar to the other concentrations (i.e., 1 and $5 \mathrm{~mol} \%$ ). It can be deduced from these behaviors that the interaction between the phosphate glass and the TMs cations is clearly characterized by the surface acidity of the polyanionic frameworks. It means, for example, that the electron transfer between the cation and the framework deformation plays a significant role in the transition metal doped phosphate glass bond. The incorporation of network modifiers such as transition metals ions in the glass structure induces defects in the network due to depolymerization of the glass network, which increases the concentration of $\mathrm{Na}^{+}$ions. These results are in agreement with dielectric spectroscopy results [11]. Morinaga et al.

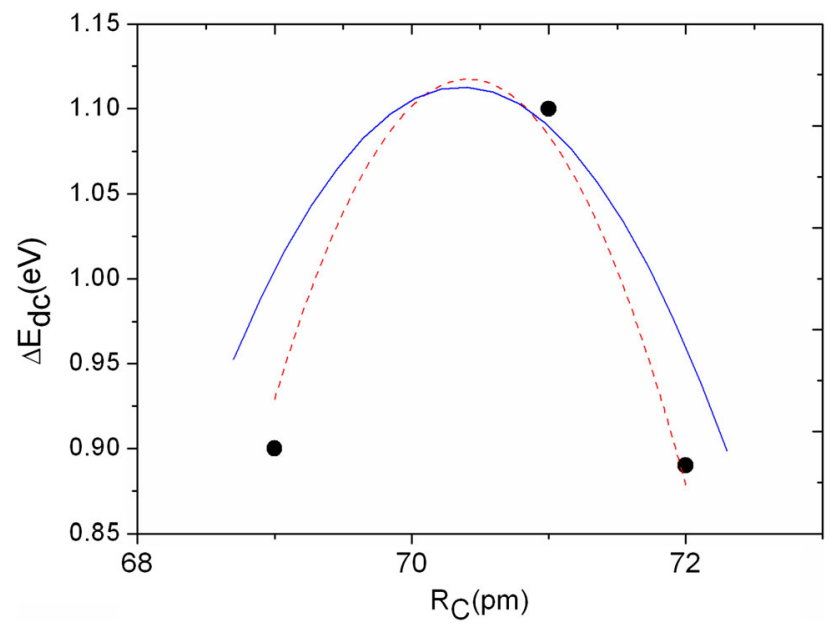

Fig. 5 Constrained fits (-) and unconstrained fits (-) obtained with Eq. (16) of the experimental (symbols) activation energy for dc conductivity for the transition-metals ions doped zinc phosphate glass (1 mol \%). The corresponding parameters $X_{i}$ constrained fit) obtained by the nonlinear least-squares fitting procedure are reported in Table 5
[32] deduced that the phosphate matrix has a higher reducing effect on the transition metal.

\section{Conclusion}

The frequency-dependent conductivity of transition metals ions doped zinc phosphate glasses is studied over a frequency range from $10^{-2} \mathrm{~Hz}$ to $1 \mathrm{MHz}$ under isothermal condition from 273 to $473 \mathrm{~K}$ by alternating current impedance spectroscopy. Furthermore, a microscopic model, with a few approximations, based on density functional theory principles, is proposed and favorably compared to the experimental data extracted from the dc activation energy of a cation out of its site. However, the different behaviors are explained qualitatively and account for the nature of the dominant interactions between the phosphate glass network and the transition metal ions. Noticeably, the results show a monotonous evolution of the activation energy of the dc conductivity, determined on phosphate glasses doped with transition metals ions, which reveals preponderance of the covalent energy term.

Open Access This article is licensed under a Creative Commons Attribution 4.0 International License, which permits use, sharing, adaptation, distribution and reproduction in any medium or format, as long as you give appropriate credit to the original author(s) and the source, provide a link to the Creative Commons licence, and indicate if changes were made. The images or other third party material in this article are included in the article's Creative Commons licence, unless indicated otherwise in a credit line to the material. If material is not included in the article's Creative Commons licence and your intended use is not permitted by statutory regulation or exceeds the permitted use, you will need to obtain permission directly from the copyright holder. To view a copy of this licence, visit http://creativecommons.org/licenses/by/4.0/.

\section{References}

[1] M T Amarkumar, T Sankarappa, P Sujatha, G B Abdul Azeem and K Devidas Santoshkumar Mater. Today Proc. 261960 (2020).

[2] Y Shahmoradi and D Souri Ceram. Int. 457857 (2019).

[3] A M Abdelghany, G El-Damrawi, A H Oraby and M A Madshal Physica B Condens. Matter. 57322 (2019).

[4] D Souri, M Elahi and M S Yazdanpanah Cent. Eur. J. Phys. 6 306 (2008).

[5] N F Mott Adv. Phys. 1649 (1967).

[6] D Souri, F Honarvar and Z E Tahan J. Alloys Compd. 699601 (2017).

[7] I G Austin and N F Mott Adv. Phys. 1841 (1969).

[8] N A Deskins and M Dupuis Phys. Rev. B75 195212 (2007).

[9] D Souri, I G Austin, P Azizpour and H Zaliani J. Electron. Mater. 439 (2014).

[10] M Sayer and A Mansingh Phys. Rev. B 612 (1972).

[11] C Kalai, M Kharroubi, S Balme, A Belbel and F Lalam Glass Phys. Chem. 66472 (2019).

[12] L A Dissado and R M Hill Philos. Mag. B 41625 (1980). 
[13] A K Jonscher J. Mater. Sci. 13553 (1978).

[14] R J Barczynski and L Murawski J. Non Cryst. Solids 3071055 (2002).

[15] P Hohenberg and W Kohn Phys. Rev. B 136864 (1964).

[16] R G Parr and W Yang Annu. Rev. Phys. Chem. 46701 (1995).

[17] W J Mortier Struct. Bond. (Berlin) 66125 (1987).

[18] R F Nalewajski and R G Parr J. Chem. Phys. 77399 (1982).

[19] R G Parr and W Yang Density Functional Theory of Atoms and Molecules. (New York: Oxford University Press) (1989)

[20] W J Mortier, S K Ghosh and S Shankar ibid. 1084315 (1986).

[21] G Maurin, P Senet, S Devautour, F Henn and J C Giuntini $J$. Chem. Phys. 1171405 (2002).

[22] P Senet J. Chem. Phys. 1056471 (1996).

[23] R G Parr and R G Pearson J. Am. Chem. Soc. 1057512 (1983).

[24] R F. Nalewajski J. Phys. Chem. 892831 (1985)

[25] J C Giuntini, G Maurin, S Devautour, F Henn and J V Zanchetta J. Chem. Phys. 1134498 (2000).
[26] L Anderson and D A Stuart J. Am. Ceram. Soc. 37573 (1954).

[27] R G Pearson Inorg. Chem. 27734 (1988).

[28] G O A Janssens, B G Baekelandt, H Toufar, W J Mortier and R A Schoonheydt J. Phys. Chem. 993251 (1995).

[29] R T Sanderson Chemical Bonds and Bond Energy. (New York: Academic Press) (1976)

[30] M Aniya Solid State Ion. 79259 (1995).

[31] J E Huheey, E A Keiter and R L Keiter Inorganic Chemistry: Principles of Structure and reactivity, (New York: Haper Collins College Publishers) p. 114-115 (1993)

[32] K Morinaga, H Yoshida and H Takebe J. Am. Ceram. Soc. 7712 3113 (1994).

Publisher's Note Springer Nature remains neutral with regard to jurisdictional claims in published maps and institutional affiliations. 